\title{
PERANAN 3D AXIAL HYPERCUBE T2 FAT SAT PADA PEMERIKSAAN MRI PELVIS DENGAN KASUSU FISTULA PERIANAL
}

\author{
${ }^{1}$ Istiqomah Nur'aini, ${ }^{2}$ Nuzul Iman, ${ }^{3}$ Eunike Serfina Fajarini \\ ${ }^{1.2}$ Departemen Radiologi RS EMC Tangerang \\ ${ }^{3} G E$ Healthcare Indonesia \\ Corresponding author: Istiqomah Nur'aini \\ Email: istiiqomah_nuraini@yahoo.com
}

\begin{abstract}
Background: Fistula is an abnormal relationship between two berepitel places. Fistula ani is a fistula that connects the anal canal to the skin around the anus (or to other organs such as the vagina). Magnetic Resonance Imaging (MRI) Pelvis is one of the right modalities to describe anatomical abnormalities of the pelvis, with the selection of appropriate protocol sequences so that informative MRI images are obtained.

Method: This research is a qualitative detiptive study using a sample of 5 patients, with the same complaints, there are lumps such as ulcers in the anus area and there are eyes that secrete fluids such as pus and without a previous medical history. Mri Pelvic examination without contrast using MRI 1.5 Tesla Signa Explorer GE brand in Radiology RS EMC Tangerang. Taking a T2 axial sekuen to see the entire pelvis. Focus on perianal fistula area with sagittal sekuen $\mathrm{T} 2$, sagittal $\mathrm{T} 2$ fat sat, axial T2, axial T2 fat sat, coronol T2, coronal T2 fat sat and 3D axial hypercube T2 fat sat.

Results: Sagittal T2 fat sat, axial T2 fat sat, and coronal T2 fat sat appear anatomically from perianal fistula and other abnormalities around them. In 3D axial hypercube $\mathrm{T} 2$ fat sat obtained anatomical picture of perianal fistula with thinner slice thickness.

Conclusion: Mri Pelvis without contrast is a very accurate diagnostic imajing in showing perianal fistula abnormalities with a clear anatomical picture, is a non-inspative examination. Uses axial T2 sekuen for the entire pelvis. Sagittal T2, sagittal T2 fat sat, axial T2, axial T2 fat sat, coronal T2, coronal T2 fat sat, 3D axial hypercube $\mathrm{T} 2$ fat sat focus on perianal fistula area.
\end{abstract}

Keywords: MRI Pelvis; Fustula Anal; Parameter

\section{Pendahuluan}

Fistula adalah hubungan abnormal antara dua tempat berepitel. Fistula ani adalah adalah fistula yang menghubungkan antara kanalis anal ke kulit di sekitar anus (ataupun ke organ lain seperti ke vagina) (Emerson Budiarman Masli, 2012).

Pada permukaan kulit bisa terlihat satu atau lebih lubang fistula, dan dari lubang fistula tersebut dapat keluar nanah ataupun kotoran saat buang air besar (Emerson Budiarman Masli, 2012).

Fistula ani sering terjadi pada laki laki berumur 20-40 tahun, berkisar 1-3 kasus tiap 10.000 orang. Sebagian besar fistula terbentuk dari sebuah abses (tapi tidak semua abses menjadi fistula). Sekitar $40 \%$ pasien dengan abses akan terbentuk fistula (Emerson Budiarman Masli, 2012).

Fistula ani juga dapat terjadi pada pasien dengan kondisi inflamasi berkepanjangan pada usus, seperti pada Irritable Bowel Syndrome (IBS), diverticulitis, colitis ilseratif, dan penyakit crohn, kanker rektum, tuberkulosis usus, HIV-AIDS dan infeksi lain pada daerah ano-rektal (Emerson Budiarman Masli, 2012).

Sebagian besar fistula ani memerlukan operasi karena fistula ani jarang sembuh secara spontan. Setelah operasi resiko kekambuhan fistula ani termasuk cukup tinggi sekitar $21 \%$ (satu dari lima pasien dengan fistula ani post operasi akan mengalami kekambuhan) (Emerson Budiarman Masli, 2012).

MRI menjadi modalitas penting dalam diagnosis fistula perianal, sebelum dilakukan nya tindakan operasi karena pemeriksaan MRI dapat dilakukan tanpa tindakan inpasif dan dirasa lebih nyaman oleh pasien karena pemeriksaan MRI tidak langsung bersentuhan dengan fistula anal tersebut.

Anus adalah bagian terakhir dari saluran pencernaan. Panjang anus adalah kira-kita $4-5 \mathrm{~cm}$. Anus memainkan peranan penting untuk defekasi. Sekiranya terjadi kelainan, defekasi tidak dapat 
berlangsung normal. Terdapat beberapa otot yang membantu anus agar defekasi lancar seperti

\section{Metode}

Penelitian ini merupakan penelitian desktiptif kualitatif dengan 5 sampel pasien, terdiri dari 4 orang pasien laki-laki dan 1 orang pasien perempuan dengan rentang usia sekitar 30-50 tahun. Data diambil antara Oktober 2019 hingga September 2020. Area pemeriksaan MRI adalah di daerah pelvis seluas daerah fistula perianal.

Pemeriksaan MRI pelvis dilakukan di Radiologi RS EMC Tangerang dengan modalitas MRI Signa Explorer 1,5 Tesla merk GE menggunakan koil Body Array. Pemilihan sekuen dalam pemeriksaan ini adalah Axial T2 untuk melihat keseluruhan pelvis, dan pengambilan pada daerah fistula perianal dengan sekuen Sagittal T2, Sagittal T2 fat sat, Axial T2, Axial T2 fat sat, Coronal T2, Coronal T2 fat sat dan 3D Axial Hypercube T2 fat sat.

\section{Hasil dan Pembahasan}

Di RS EMC Tangerang, pemeriksaan MRI Pelvis Non Kontras dengan Klinis Fistula Ani tidak diperlukan adanya persiapan pasien.

Keuntungan dari pencitraan MRI termasuk pencitraan multi-planar serta menghasilkan gambaran jaringan lunak dengan kualitas tinggi sehingga dapat memperlihatkan jalur fistula dalam kaitannya dengan organ lain. Pemeriksaan MRI memberikan detail anatomi yang sangat baik dari sfingter ani dan batas anatomi panggul.

Pertama-tama kami melakukan pemindaian sekuen axial T2 propeller untuk memperlihatkan gambaran perineum dan digunakan sebagai guiding untuk membuat sekuen sagittal T2 (seluas area fistula perianal). Selanjutnya dilakukan pemindaian sekuen sagittal T2 fat sat (seluas area fistula perianal) yang digunakan sebagai guiding sekuen lainnya khusus daerah fistula.

Berikut urutan sekuen yang kami buat:

1. Pelvic overview: Axial T2 Propeller

2. Fistula perianal region:

a. T2 FSE ( Sagiytal, axial, coronal)

b. T2 FSE Fat Sat (Axial, sagittal, coronal)

c. Ax 3D Hypercube T2 Fat Sat

Total waktu pemeriksaan yang dibutuhkan untuk membuat seluruh sekuen diatas adalah 20 menit. m.puborektal.

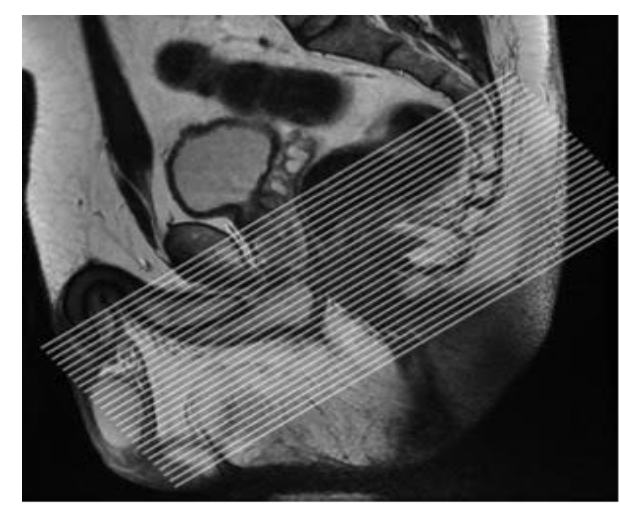

(a)

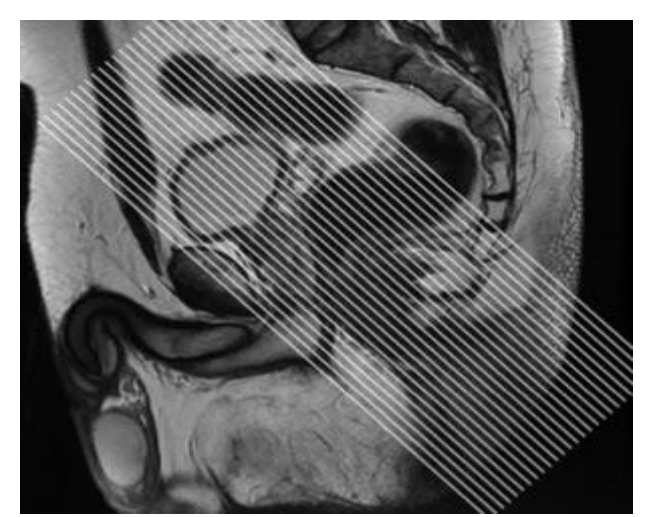

(b)

Gambar 1. (a) Suggested orientation for axial $M R$ imaging of the anal canal. Sagittal T2W image through the midline is used to obtain image that area truly axial relative to the anal canal. (b) Suggested orientation for axial MR imaging of the anal canal. Coronal MR imaging is performed at $90^{\circ}$ relative to the axial plane to obtain image parallel to the long axis of the anal canal.

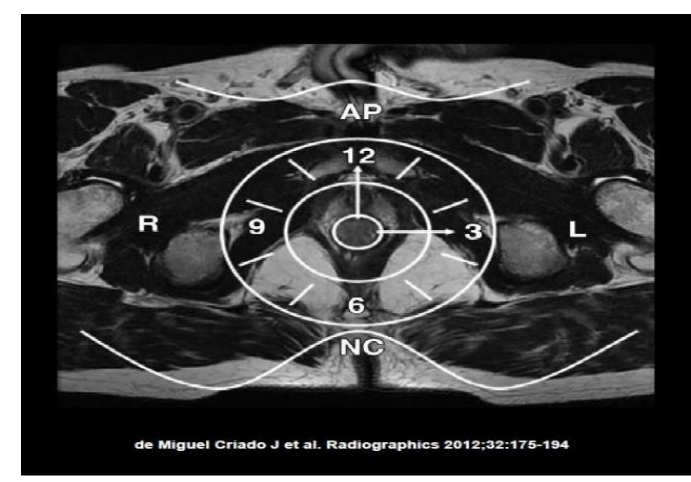

Gambar 2. Anal clock. Axial T2W MR image of the male perineum shown the anal clock diagram used to correctly locate anal fistulas with respect into the anal canal. $A P=$ anterior perineum, $L=$ left aspect of the anal canal, $N C=$ natal cleft, $R=$ right aspect ot the canal. 
Tabel 1: Protokol pemeriksaan MRI Pelvis pada kasus fistula anal menggunakan MRI Signa Explorer 1,5 Tesla merk GE dengan menggunakan coil Body Array

\begin{tabular}{|c|c|c|c|c|c|c|c|c|}
\hline Parameter & Axial & Sagital & Sagital & Coronal & Axial & Axial & Coronal & Axial \\
\hline Pulse sequence & $\mathrm{T} 2$ & $\mathrm{~T} 2$ & T2 Fat Sat & T2 Fat Sat & T2 Fat Sat & $\mathrm{T} 2$ & $\mathrm{~T} 2$ & $\begin{array}{c}\text { 3D } \\
\text { HYPERCUBE } \\
\text { AX T2 Fat Sat }\end{array}$ \\
\hline TR (ms) & 3161 & 3452 & 3387 & 2895 & 5032 & 7779 & 4288 & 2000 \\
\hline TE (ms) & 80 & 85 & 75 & 70 & 70 & 120 & 85 & 91 \\
\hline \multicolumn{9}{|l|}{$\mathrm{TI}(\mathrm{ms})$} \\
\hline BW & 41,67 & 50 & 50 & 41,67 & 50 & 41,67 & 50 & 31,25 \\
\hline ETL & 18 & 20 & 18 & 21 & 21 & 23 & 20 & 130 \\
\hline NEX & 1 & 2 & 2 & 2,5 & 3 & 1 & 1 & 4 \\
\hline FOV & 38 & 28 & 28 & 28 & 28 & 28 & 38 & 36 \\
\hline \multicolumn{9}{|l|}{ Matrix } \\
\hline Slice/gap (mm) & $5,0-2,0$ & $3,5-1,0$ & $3,5-1,0$ & $3,5-1,0$ & $3,5-1,0$ & $3,5-1,0$ & $3,5-1,0$ & 2 \\
\hline Flip Angel ( ()$^{\circ}$ & 160 & 160 & 160 & 160 & 160 & 180 & 160 & \\
\hline Scan time (s) & 1,48 & 2,49 & 2,46 & 2,31 & 2,36 & 2,44 & 1,47 & 3,37 \\
\hline Slice & 29 & 20 & 20 & 29 & 29 & 29 & 29 & 256 \\
\hline Frek direction & $\mathrm{A} / \mathrm{P}$ & $\mathrm{A} / \mathrm{P}$ & A/P & $R / L$ & $R / L$ & $R / L$ & $\mathrm{R} / \mathrm{L}$ & $\mathrm{S} / \mathrm{I}$ \\
\hline
\end{tabular}

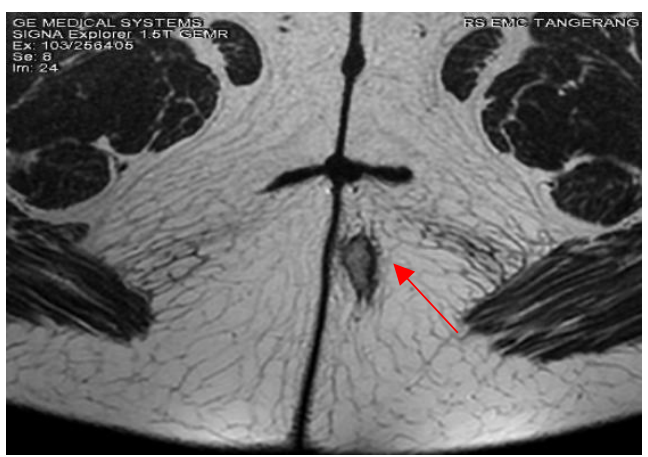

(a)

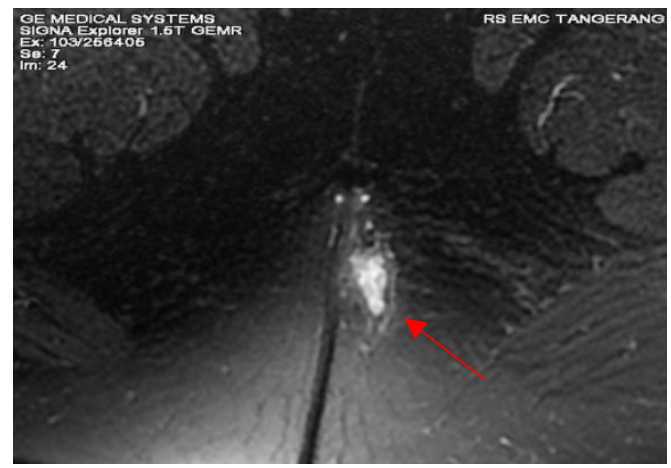

(b)

Gambar 16. (a) Axial $T 2$ tanda panah menunjukan gambaran Intre-sphincteric Fistula (b) Axial T2 fat sat tanda panah menunjukan gambaran Intre-sphincteric Fistula (Sumber : RS EMC Tangerang, 2020)

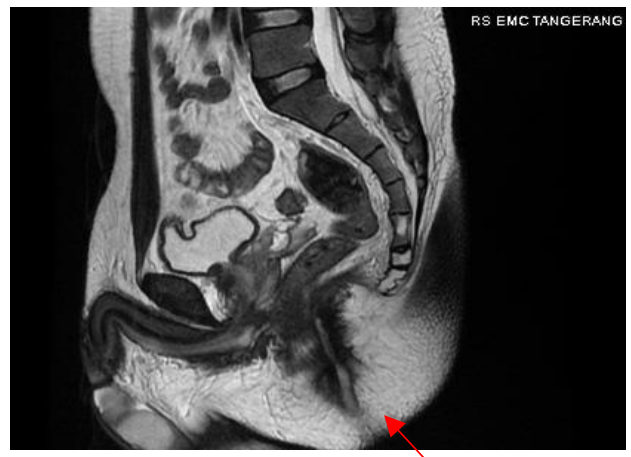

(a)

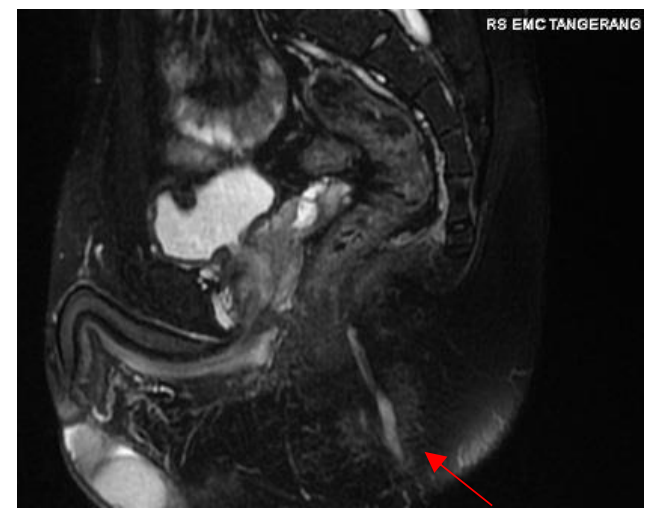

(b)

Gambar 16. (a) Sagittal $T 2$ tanda panah menunjukan gambaran Intre-sphincteric Fistula (b) Sagittal T2 fat sat tanda panah menunjukan gambaran Intre-sphincteric Fistula. (Sumber : RS EMC Tangerang, 2020)

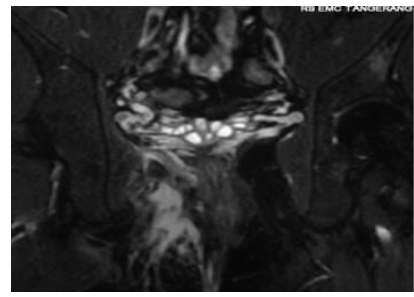

(a)

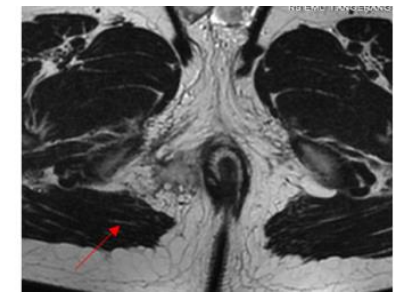

(b)
Gambar 17. (a) Coronal T2 fat sat tanda panah menunjukan adanya abses regio ischio-anal dextra (b) Axial T2 tanda panah menunjukan adanya abses regio ischio-anal dextra. (Sumber : RS EMC Tangerang, 2020) 


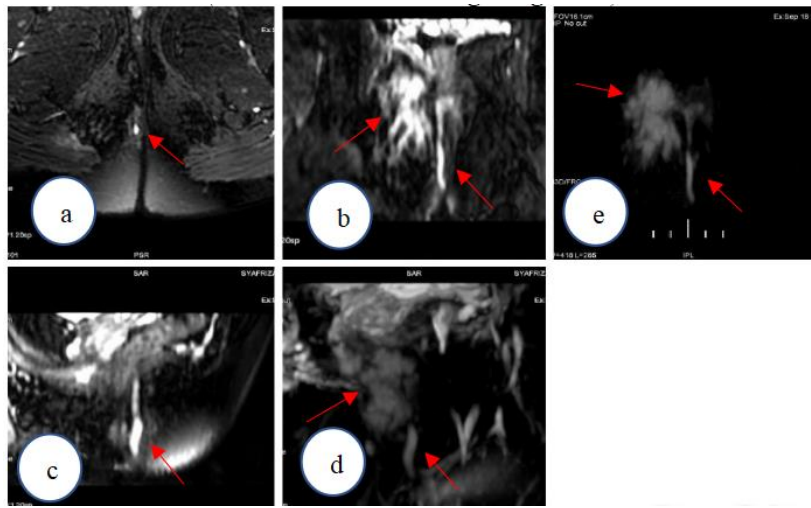

Gambar 18. Menunjukan tampilan sequen $3 D$ axial hypercube $T 2$ fat sat pada software reformat (a) $3 D$ axial hypercubeT2 fat sat tanda panah menunjukan gambaran fistula anal (b) potongan coronal tanda panah menunjukan ada nya abses dan fistula anal (c) potongan sagittal tanda panah menunjukan fistula anal (d) gambaran MIP tanda panah menunjukan ada nya abses dan fistula anal (e) hasil reformat $3 D$ axial hypercube T2 fat sat tanda panah menunjukan abses dan fistula anal

Teknik pencitraan 3D memiliki beberapa keunggulan dibandingkan pencitraan 2D, antara lain : Tidak ada ketergantungan operator dalam memperoleh gambar dalam kemiringan tertentu, area scan dapat diperbesar (sesuai luas kelainan), dapat memperoleh gambaran yang lebih tipis (sehingga kita dapat mengamati perjalanan fistula tersebut, apakah ada interaksi dengan organ lain atau tidak), menghasilkan signal-to-noise yang lebih tinggi, dan waktu pencitraan dapat dikurangi (sangat penting untuk pasien yang tidak kooperatif).

Pencitraan MRI memiliki peran penting dalam membantu menentukan perawatan yang tepat untuk fistula perianal karena strategi perawatan harus disesuaikan dengan jenis fistula perianal dan tingkat keterlibatan struktur panggul di sekitarnya.

Pada pemeriksaan MRI, jenis dan lokasi seluruh saluran fistula primer, saluran fistula sekunder, horse shoe, abses, ekstensi pada fistula supralevator dan fistula yang berhubungan dengan organ lain dapat terlihat, hal ini sangat penting untuk klasifikasi dan pengobatan fistula. Penilaian fistula yang kurang tepat dapat menyebabkan fistula sederhana berkembang menjadi fistula kompleks.

\section{Simpulan}

Pemeriksaan MRI dapat digunakan untuk evaluasi pra operasi fistula perianal, memberikan hasil akurat, cepat, dan non-inpasif untuk melakukan penilaian pra-bedah. Untuk menghasilkan gambaran yang tipis dan informatif dapat dilakukan dengan teknik pemeriksaan 3D.

Pemeriksaan MRI dapat menggambarkan jalur fistula yang tepat dan hubungannya dengan organ disekitarnya, serta dapat menggambarkan fistula atau abses sekunder.

Sekuen yang kami buat untuk pemeriksaan MRI Pelvis pada kasus fistula anal, antara lain: Axial T2 Propeller (Pelvis overview), Sagittal T2, Sagittal T2 fat sat, Axial T2, Axial T2 fat sat, Coronal T2, Coronal T2 fat sat dan 3D Axial Hypercube T2 fat sat (focus pada daerah fistula anal). Total waktu pemeriksaan untuk membuat seluruh sequen diatas kurang lebih 19 menit.

\section{Daftar Pustaka}

Beckingham IJ, Spencer JA, Ward J, Dyke GW, Adams C, Ambrose NS. Prospective evaluation of dynamic contrast enhanced magnetic resonance imaging in the evaluation of fistula in ano. Br J Surg 1996;83(10):13961398.

Buchanan G, Halligan S, Williams A, et al. Effect of MRI on clinical outcome of recurrent fistulain-ano. Lancet 2002;360(9346):1661-1662.

Chiari H. Uber die nalen divertikel der rectumshleimhaut undo ihre beziehung zu den anal fisteln. Wien Med Press 1878;19:14821483.

Hori M, Oto A, Orrin S, Suzuki K, Baron RL. Diffusion-weighted MRI: a new tool for the diagnosis of fistula in ano. J Magn Reson Imaging 2009;30(5): 1021-1026.

Horsthuis K, Lavini C, Bipat S, Stokkers PC, Stoker J. Perianal Crohn disease: evaluation of dynamic contrast-enhanced MR imaging as an indicator of disease activity. Radiology 2009;251(2):380-387.

Lichy MP, Wietek BM, Mugler JP 3rd, et al. Magnetic resonance imaging of the body trunk using a single-slab, 3-dimensional, T2weighted turbo-spin-echo sequence with high sampling efficiency (SPACE) for high spatial resolution imaging: initial clinical experiences. Invest Radiol 2005;40(12): 754-760.

McColl I. The comparative anatomy and pathology of anal glands. Arris and Gale lecture delivered at the Royal College of Surgeons of England on 25th February 1965. Ann R Coll Surg Engl 1967;40(1):36-37.

Morris J, Spencer JA, Ambrose NS. MR imaging classification of perianal fistulas and its 
implications for patient management. RadioGraphics 2000;20(3):623-635; discussion 635-637.

Parks AG. Pathogenesis and treatment of fistula-inano. BMJ 1961;1(5224):463-469.

Proscia N, Jaffe TA, Neville AM, Wang CL, Dale BM, Merkle EM. MRI of the pelvis in women: 3D versus 2D T2-weighted technique. AJR Am J Roent-genol 2010;195(1):254-259

Seow-Choen, Phillips RK. Insights gained from the management of problematical anal fistulae at St. Mark's Hospital, 1984-88. Br J Surg 1991;78(5): 539-541.

Spencer JA, Ward J, Beckingham IJ, Adams C, Ambrose NS. Dynamic contrast-enhanced MR imaging of perianal fistulas. AJR Am J Roentgenol 1996;167 (3):735-741.

Schaefer O, Lohrmann C, Langer M. Assessment of anal fistulas with high-resolution subtraction MR-fistulography: comparison with surgical findings. J Magn Reson Imaging 2004;19(1):91-98. 